\title{
Paraneoplastic encephalitis associated with renal cell carcinoma
}

\author{
Yoonhyuk Jang, SeonDeuk Kim, Kon Chu, Sang Kun Lee, Soon-Tae Lee \\ Department of Neurology, Seoul National University Hospital, Seoul, Korea
}

\section{Purpose}

Paraneoplastic encephalitis is autoimmune encephalitis accompanied by tumors. Renal cell carcinoma (RCC) is a kidney cancer originating from various types of renal cells and rarely has been associated with paraneoplastic neurologic manifestation. We identified a case series of paraneoplastic encephalitis-associated RCC.

\section{Methods}

From a prospective institutional cohort, we identified autoimmune encephalitis patients with RCC. The association between RCC and encephalitis was determined by the following criteria: (1) possible autoimmune encephalitis according to the operational autoimmune encephalitis diagnostic criteria and (2) RCC simultaneously diagnosed with neurological manifestation of encephalitis.

\section{Results}

A total of three patients presented encephalitis accompanied by RCC. Two patients had clear cell RCC, and one had chromophobe RCC. All patients showed limbic encephalitis with cognitive decline, memory impairment, or seizure. Brain magnetic resonance imaging showed T2 hyperintensities at the mesial temporal lobe in two patients with clear cell RCC but no remarkable findings in one patient with chromophobe RCC. While one patient who had early surgery within one month of RCC onset had a favorable response to the treatment, the other two patients showed a partial response and a detrimental result.

\section{Conclusion}

Paraneoplastic encephalitis associated with RCC presented as limbic encephalitis and was responsive to immunotherapy combined with tumor resection. As our cases identified, RCC should be considered as a cause of seronegative autoimmune encephalitis.

Keywords: Encephalitis, Paraneoplastic limbic, Renal cell carcinoma, Nervous system paraneoplastic syndromes, Rare cancer

\section{INTRODUCTION}

Paraneoplastic encephalitis is a neurologic manifestation of paraneoplastic syndrome that can present as seizure, memory impairment, cognitive decline, behavior changes, gait disturbance, and/or psychiatric symptoms [1]. Classically, paraneoplastic encephalitis has been defined as the presence or high risk of cancer accompanied by certain neurologic symptoms including limbic encephalitis, encephalomyelitis, subacute cere- bellar degeneration, and subacute sensory neuropathy, without any other causes [2]. Recent studies have found various autoantibodies associated with tumors [3]. Certain types of tumors, such as small-cell lung carcinoma and thymoma, are more likely to develop into paraneoplastic encephalitis. However, there are few reports that paraneoplastic encephalitis can occur in other cancers [4-7]. In such cases, paraneoplastic encephalitis is severe enough to complicate medical decisions.

\footnotetext{
Received: March 27, 2021 Revised: April 17, 2021 Accepted: April 23, 2021

Correspondence: Soon-Tae Lee

Department of Neurology, Seoul National University Hospital, 101 Daehak-ro, Jongno-gu, Seoul 03080, Korea

E-mail: staelee@snu.ac.kr

ORCID: https://orcid.org/0000-0003-4767-7564
}

Copyright (C) 2021 by The Korean Encephalitis and Neuroinflammation Society

This is an open access article distributed under the terms of the Creative Commons Attribution Non-Commercial License (http://creativecommons.org/licenses/by-nc/4.0/) which permits unrestricted non-commercial use, distribution, and reproduction in any medium, provided the original work is properly cited. 
Renal cell carcinoma (RCC) is cancer developed from renal cells of several different types according to the cellular origin [8]. Clear cell is the most common type of RCC, though few case reports of paraneoplastic encephalitis associated with clear cell RCC have been published [9-11]. All aforementioned cases presented with limbic encephalitis, showing memory impairment and cognitive decline without clear lesion on brain magnetic resonance imaging (MRI). However, a limited number of case studies fully characterized the clinical phenotype and outcomes of paraneoplastic encephalitis associated with RCC.

Here, we identified three patients who had autoimmune encephalitis accompanied by RCC and reported the clinical characteristics, treatments, and outcomes.

\section{METHODS}

We enrolled patients with encephalitis accompanied by RCC from our prospective cohort of autoimmune encephalitis between September 2019 and February 2021. The patients who fulfilled the following criteria were included as paraneoplastic encephalitis associated with RCC: (1) possible autoimmune encephalitis according to the operational autoimmune encephalitis diagnostic criteria [1] and (2) RCC simultaneous diagnosis with neurological manifestation of encephalitis. We confirm that we have read the Journal's position on issues involved in ethical publication and affirm that this report is consistent with those guidelines. This study was approved by the Seoul National University Hospital Institutional Review Board (1705-130-856). We received written informed consent from all patients and/or legal guardians.

We performed a diagnostic approach following specific guidelines of the Korean Epilepsy Society for patients with unknown encephalitis and epilepsy type [12]. Laboratory examinations included tests for viral polymerase chain reaction (PCR), tuberculosis PCR, bacterial and fungal microbial culture, neurosarcoidosis, and neuro-Behçet. We screened for autoantibodies including $N$-methyl-D-aspartate receptor, leucine-rich glioma-inactivated 1 , aquaporin 4 , myelin oligodendrocyte glycoprotein, myelin-associated glycoprotein, contactin-associated protein-like 2, anti- $\alpha$-amino-3-hydroxy-5-methyl-4-isoxazolepropionic acid 1, AMPA2, and $\gamma$-aminobutyric-acid type B receptors, $\mathrm{Hu}$, Yo, Ri, Ma2, CV2/CRMP5, amphiphysin, recoverin, Sox1, and Titin (Euroimmun AG, Lubeck, Germany). Cancer screening was performed with low-dose chest computed tomography (CT), abdominal and pelvic CT, and stool hemoglobin. Mammography and breast sonography were performed additionally in female patients.

Empirical immunotherapy was administrated as soon as possible if autoimmune etiology was suspected. Based on cancer stage, patients were assigned to radical or partial nephrectomy as the primary treatment for RCC. Clinical outcomes were assessed with the Clinical Scale in Autoimmune Encephalitis (CASE) [13] and modified Rankin scale (mRS).

\section{RESULTS}

Characteristics and treatment outcome of patients Among 558 patients who were enrolled in our prospective cohort, three presented with autoimmune encephalitis and RCC (Table 1). All showed limbic encephalitis, presenting with altered mentality, memory impairment, or seizure. Two patients (patient 1 and 2) had clear cell RCC, and one patient (patient 3) had chromophobe RCC. The cancer was detected before neurological manifestation in two patients (patient 1 and 3); in patient 2 , the RCC was found during malignancy workup for autoimmune encephalitis. For the autoantibody test, two patients (patient 1 and 3) showed nonspecific antibodies such as microsome antibody and antinuclear antibody (ANA); however, none had classic paraneoplastic autoantibodies such as anti-Hu, Yo, Ri, Ma2, CV2/CRMP5, amphiphysin, recoverin, Soxl, or Titin. In brain MRI, patients with clear cell RCC (patient 1 and 2) showed T2 hypersignal intensities (HSI) involving the mesial temporal lobe, and the patient with chromophobe RCC (patient 3) had no remarkable findings. All patients received immunotherapy and nephrectomy. Two patients (patient 1 and 3) underwent radical nephrectomy, and one (patient 2) had partial nephrectomy. However, only one patient (patient 1) had early surgery within one month of onset of neurological symptoms. While patient 1 had a favorable response to the treatment, patient 2 had a partial response with sequelae, and patient 3 showed poor results without clear improvement. The clinical histories of the three patients are described below.

\section{Case 1 (patient 1)}

A 54-year-old female was referred to our hospital due to altered mental state and memory impairment. One month before neurologic manifestation, she underwent cancer screening due to progressive body weight loss (9 kg over 2 years), and a $2.0-\mathrm{cm}$ sized right upper kidney mass was detected by abdominal CT (Figure 1A). While waiting for admission for surgery, the patient presented fever, confusion, and global amnesia. She received empiric antibiotic and antiviral treatment under diagnosis of infectious meningoencephalitis at a local hospital, but the global 
Table 1 Characteristics of patients with renal cell carcinoma-associated encephalitis

\begin{tabular}{|c|c|c|c|}
\hline Characteristic & Patient 1 & Patient 2 & Patient 3 \\
\hline Age $(y r) /$ sex & 54/female & $51 / \mathrm{M}$ & $54 / F$ \\
\hline Underlying disease & $\begin{array}{l}\text { HTN, dyslipidemia, major depressive } \\
\text { disorder }\end{array}$ & Previously healthy & HTN, diabetes mellitus \\
\hline Clinical symptom & $\begin{array}{l}\text { Fever, altered mentality, global } \\
\text { amnesia }\end{array}$ & $\begin{array}{l}\text { Fever, left facial focal tonic onset to } \\
\text { bilateral generalized tonic seizure, } \\
\text { characteristic change }\end{array}$ & $\begin{array}{l}\text { Generalized edema, transient } \\
\text { right-sided facial palsy and weakness, } \\
\text { memory impairment, aphasia, } \\
\text { dysarthria, visual hallucination }\end{array}$ \\
\hline $\begin{array}{l}\text { Renal cell carcinoma side/type/size/ } \\
\text { stage/grade }\end{array}$ & $\begin{array}{l}\text { Right clear cell RCC, } 2.2 \times 2.0 \times \\
2.0 \mathrm{~cm}^{3} \text {, T1aNOMO, WHO grade II }\end{array}$ & $\begin{array}{l}\text { Right clear cell RCC, } 2.5 \times 2.4 \times \\
2.3 \mathrm{~cm}^{3}, \text { T1aNOMO, WHO grade III }\end{array}$ & $\begin{array}{l}\text { Left chromophobe RCC, } 3.7 \times 3.0 \times \\
2.0 \mathrm{~cm}^{3}, \text { T1aNOMO, WHO grade III }\end{array}$ \\
\hline Autoantibody test & Microsome antibody (4430) & Negative & ANA (1:40), weakly positive \\
\hline CSF worst profile & $\begin{array}{l}\text { WBC2 (P1L1); Ptn, 49; Glu, 99/168; } \\
\text { IgG index, } 0.57\end{array}$ & $\begin{array}{l}\text { WBC17 (POM0017); Ptn, 24; Glu, } \\
\text { 59/109; IgG index, 0.79 }\end{array}$ & $\begin{array}{l}\text { WBC12 (P4L404); Ptn, 193; Glu, } \\
\text { 83/140 }\end{array}$ \\
\hline Brain MRI & $\begin{array}{l}\text { Symmetric T2 hyperintensities } \\
\text { involving both hippocampi }\end{array}$ & $\begin{array}{l}\text { Multifocal T2 HSI in the right } \\
\text { temporal lobe, thalamus, frontal lobe, } \\
\text { bilateral medial frontal lobes }\end{array}$ & Unremarkable findings \\
\hline Abdominal CT/MRI & $\begin{array}{l}3.0 \mathrm{~cm} \text {-sized heterogeneously } \\
\text { enhancing mass in the right kidney } \\
\text { upper pole, anterior aspect }\end{array}$ & $\begin{array}{l}2.5-\mathrm{cm} \text {-sized heterogeneously } \\
\text { enhancing mass in the upper pole of } \\
\text { the right kidney }\end{array}$ & $\begin{array}{l}\text { 3.7-cm-sized exophytic mass in the } \\
\text { upper pole of the left kidney }\end{array}$ \\
\hline \multicolumn{4}{|l|}{ Duration from neurological symptoms (wk) } \\
\hline To immunotherapy & 3 & 1 & 3 \\
\hline To surgery & 4 & 7 & 7 \\
\hline Immunotherapy & High-dose corticosteroid, IVlg & $\begin{array}{l}\text { High-dose corticosteroid, IVlg, } \\
\text { rituximab }\end{array}$ & IVlg, rituximab \\
\hline Surgery & Right radical, open nephrectomy & Right partial, robotic nephrectomy & $\begin{array}{l}\text { Left radical, laparoscopic nephrectomy } \\
\text { with en bloc adrenalectomy }\end{array}$ \\
\hline Response to treatment & Complete remission & Improvement of seizure & $\begin{array}{l}\text { Transient partial remission, but } \\
\text { eventually nonresponsive }\end{array}$ \\
\hline Remnant sequelae after treatment & None & $\begin{array}{l}\text { Focal onset aware temporal seizure } \\
\text { (visual hallucination, olfactory } \\
\text { hallucination) }\end{array}$ & Death \\
\hline CASE change & $5 \rightarrow 0$ & $4 \rightarrow 2$ & $9 \rightarrow 3 \rightarrow 19$ \\
\hline mRS change & $4 \rightarrow 0$ & $4 \rightarrow 3$ & $4 \rightarrow 1 \rightarrow 6$ \\
\hline
\end{tabular}

HTN, hypertension; RCC, renal cell carcinoma; WHO, World Health Organization; ANA, antinuclear antibody; WBC, white blood cell; Ptn, protein; Glu, glucose; IgG, immunoglobulin G; MRI, magnetic resonance imaging; HSI, high signal intensity; CT, computed tomography; IVIg, intravenous immunoglobulin; CASE, Clinical Assessment Scale in Autoimmune Encephalitis; mRS, modified Rankin scale.

amnesia aggravated over the next 3 weeks. At an examination in our hospital, her orientation and memory were impaired, showing a Mini-Mental Status Exam score of 20, CASE of 5, and mRS of 4 . A cerebrospinal fluid (CSF) test was within the normal range (white blood cells [WBCs], $2 / \mu \mathrm{L}$ [monocyte $50 \%$, lymphocyte 50\%]; protein, $49 \mathrm{mg} / \mathrm{dL}$; glucose ratio, 99/168), and the immunoglobulin $\mathrm{G}$ (IgG) index was at the upper normal limit at 0.57. Brain MRI revealed symmetric T2 HSI involving both hippocampi (Figure 1A). Through an extensive examination covering infection and autoantibody-mediated disease, the patient tested positive only for microsome antibodies. Therefore, we concluded that the encephalitis was paraneoplastic encephalitis associated with RCC. After immunotherapy with high-dose corticosteroid (methylprednisolone at 1,000 mg intravenously for 5 days) and intravenous immunoglobulin (IVIg) $(0.4 \mathrm{~g} / \mathrm{kg} /$ day for 5 days), she underwent a right-side radical open nephrectomy at day 30 from onset of neurological symptoms. The RCC was clear cell type, and the stage was TlaN0M0 with World Health Organization (WHO) grade II (Figure 1A). During the 1-year follow-up, her mental status and memory fully recovered without recurrence.

\section{Case 2 (patient 2)}

A 51-year-old male was admitted because of a mid-age newly-onset seizure. He was previously healthy and had no recent history of travel or vaccination. At 4 days before admission, he experienced autonomous pilomotor movement on the left side of his body for 1 minute. On the day of admission, a focal seizure originated from the left side of his facial muscles and spread to the left arm and leg. The seizure propagated into a generalized tonic seizure of 1 minute. On neurologic examination, he was drowsy and disoriented, showing CASE of 4 and mRS of 4 . Brain MRI revealed multifocal T2 HSI in the right temporal lobe, thal- 

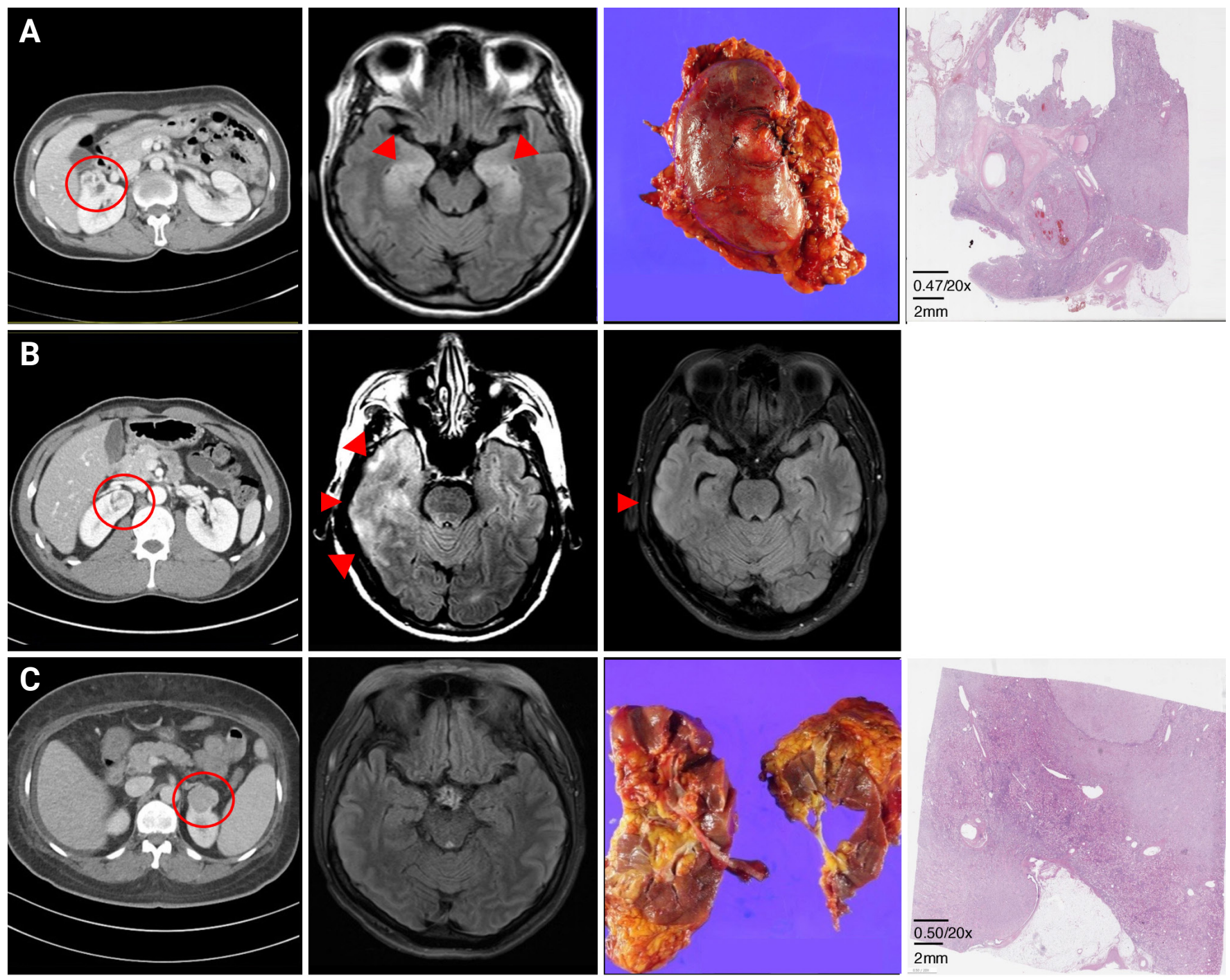

(A) Patient 1. Abdominal computed tomography (CT) showed a 3.0-cm-sized heterogeneously enhancing mass in the anterior aspect of the right kidney upper pole. Brain magnetic resonance imaging (MRI) before immunotherapy revealed symmetric T2 high signal intensities (HSI) in both hippocampi. Right radial nephrectomy was conducted, and pathologic findings showed clear cell type (H\&E). (B) Patient 2. On malignancy screenings, abdominal CT showed a 2.5-cm-sized heterogeneously enhancing mass in the right kidney upper pole. T2 HSI were seen in both hippocampi on brain MRI before treatment. After immunotherapy combined with partial nephrectomy, brain MRI revealed improvement, but the subcortical lesion remained at the right temporal lobe. (C) Patient 3. A 3.7-cm-sized exophytic mass in the left kidney upper pole was found on abdominal CT. In brain MRI, no remarkable findings were seen. Left radical nephrectomy with en bloc adrenalectomy was performed, and pathologic findings showed chromophobe type (H\&E).

amus, and bilateral medial frontal lobes, with sulcal enhancement in the right frontal lobe (Figure $1 \mathrm{~B})$. The CSF test was normal (WBC, $0 / \mu \mathrm{L}$; protein, $24 \mathrm{mg} / \mathrm{dL}$; glucose ratio, 59/101), but the IgG index was high at 0.79 . Electroencephalogram (EEG) recordings revealed a focal seizure disorder originating from the right fronto-temporoparietal area. Repetitive laboratory tests for infection were all negative, and no autoantibodies were found. However, on malignancy screenings, a 2.5 -cm-sized heteroge- neously enhancing mass was detected in the upper pole of the right kidney (Figure 1B). In suspicion of paraneoplastic encephalitis associated with RCC, immunotherapy with high-dose corticosteroid (methylprednisolone at 1,000 mg intravenously for 5 days), $\operatorname{IVIg}$ ( $0.4 \mathrm{~g} / \mathrm{kg} /$ day for 5 days), and rituximab (375 mg/m² for one cycle) was administered to the patient within 1 week from seizure onset. On day 49 from the seizure, partial nephrectomy was performed. The RCC was clear cell type of stage TlaN- 
0MO, and WHO grade was III. After treatment, the EEG data were normal, and the follow-up brain MRI revealed decreased extent of $\mathrm{T} 2$ hyperintensities at the right hemisphere, but the subcortical lesion remained at the right temporal lobe (Figure 1B). A focal-aware seizure presenting as metamorphopsia and olfactory hallucination remained as sequelae with CASE of 2 and mRS of 3.

\section{Case 3 (patient 3)}

A 54-year-old female was transferred to our hospital because of memory impairment, aphasia, and psychosis. Since 2 months before admission to our hospital, she gained bodyweight of 13 $\mathrm{kg}$ with generalized edema. The patient was admitted to a local hospital, and on abdominal CT, a 3.7-cm-sized exophytic mass was found in the upper pole of the left kidney (Figure 1C). During a further workup, transient right facial palsy and rightside weakness occurred for two hours, but brain MRI and EEG could not find any specific lesion (Figure 1C). Since 2 days before transfer to our hospital, her sleep cycle and memory were impaired suddenly, and the patient experienced visual hallucination. Indicated by neurologic exam on the transfer day, she was severely disoriented and had sensory aphasia, unable to understand the doctor's direction, showing a CASE of 9. Repeat brain MRI was normal, and a large number of theta to delta waves was seen on EEG of the bilateral hemispheres. The CSF study showed mild pleocytosis with elevated protein (WBC, 12/ $\mu \mathrm{L}$ [monocyte 33\%, lymphocyte 33\%]; protein, $193 \mathrm{mg} / \mathrm{dL}$; glucose ratio, 83/140). No evidence of infectious origin was found through extensive laboratory tests, but repetitive autoimmune antibody test showed weak positivity for ANA (1:40) and increased serum IgG $(1,727)$. Therefore, an autoimmune etiology was suspected, for IVIg ( $0.4 \mathrm{~g} / \mathrm{kg} /$ day for 5 days) was administered to the patient at 3 weeks after transient right-sided facial palsy and weakness. The patient responded to the immunotherapy, recovering mRS from 4 to 1 and lowering CASE to 3 . A radical nephrectomy with en bloc adrenalectomy was performed on day 48 from onset of the neurological manifestation, and the RCC was chromophobe type of stage TlaNOM0 and WHO grade III (Figure 1C). However, since the first 5 days after surgery, her mental status rapidly aggravated to stupor. Despite additional immunotherapy of IVIg and rituximab, the patient remained unresponsive and passed away on day 67 from onset because of multiple complications of acute renal failure and septic shock.

\section{DISCUSSION}

In this case series, we reported three patients with encephalitis accompanied by RCC. The encephalitis manifested as limbic encephalitis in all patients. Two patients with clear cell RCC had favorable outcomes of combined immunotherapy and nephrectomy, while one patient with chromophobe RCC showed a transient response but eventually a detrimental outcome. These cases suggest that RCC can present as paraneoplastic encephalitis and be responsive to immunotherapy but can have complications throughout the course of treatment.

Our patients' encephalitis accompanied by RCC could be considered paraneoplastic encephalitis. According to the former diagnostic criteria, limbic encephalitis, which our patients manifested, is a classical paraneoplastic encephalitis syndrome of the central nervous system, and our cases are consistent with the category of definite paraneoplastic encephalitis regardless of presence of onconeuronal antibodies [2]. Moreover, the recent diagnostic criteria for autoimmune encephalitis were met in all patients [1], corresponding with the definition of paraneoplastic encephalitis as autoimmune encephalitis in which immune cells sensitize a self-antigen of the nervous system in a tumor microenvironment. Therefore, immunotherapy combined with tumor resection could have been effective in our patients.

For patient 3, paraneoplastic encephalitis was accompanied by chromophobe RCC but not by clear cell RCC. To date, the cancer type paraneoplastic encephalitis associated with RCC was clear cell (Table 2). RCC is represented by a collection of heterogeneous histology, and clear cell RCC is the most common type, which originates from atypical neoplasm of a proximal convoluted tubule epithelium. Chromophobe RCC is differentiated histologically and molecularly from clear cell RCC in that it originates from the distal convoluted tubule epithelium $[8,14,15]$. Therefore, the pathomechanism of paraneoplastic encephalitis

Table 2 Review of historical cases with paraneoplastic limbic encephalitis associated with RCC

\begin{tabular}{|c|c|c|c|c|c|c|}
\hline Study & Year & No. of cases (age/sex) & Clinical manifestation & RCC type (size) & Treatment & Prognosis \\
\hline Bell et al. [9] & 1988 & 1 (49 yr/female) & Memory loss, seizure & Clear cell $(4 \mathrm{~cm})$ & Radical nephrectomy & Recovered \\
\hline Harrison et al. [10] & 2015 & 1 (66 yr/female) & Visual hallucination, memory loss & Clear cell $(8 \mathrm{~cm})$ & Radical nephrectomy & Recovered \\
\hline Zhu et al. [11] & 2019 & 1 (54 yr/male) & Headache, mood fluctuation, cognitive decline & Clear cell $(5.5 \mathrm{~cm})$ & Partial nephrectomy & Recovered \\
\hline
\end{tabular}

RCC, renal cell carcinoma. 
might be different according to origin of RCC. However, we could not find any definite autoantibodies, and patient 3 had a grave outcome after surgery. In turn, neurologic manifestation of chromophobe RCC requires further investigation.

Our case series is a small study of only three patients, but it is meaningful in that we treated them according to certain guidelines in a single tertiary institution [12]. Although paraneoplastic encephalitis with RCC is a rare condition, clinicians should be aware of it and apply immunotherapy as soon as possible because the encephalitis could remain a permanent sequela. As our cases showed that paraneoplastic encephalitis with RCC could present as limbic encephalitis, malignancy screening for RCC is recommended in patients with seronegative autoimmune encephalitis.

\section{Conflicts of Interest}

Kon Chu, Sang Kun Lee, Soon-Tae Lee have been editorial board of encephalitis since October 2020. They were not involved in the review process of this original article. No other potential conflict of interest relevant to this article was reported.

\section{Funding}

Soon-Tae Lee was supported by the Lee Sueng Moon Research Fund of the Seoul National University Hospital (3020170130).

\section{Author Contributions}

Data curation: Jang Y, Kim S, LEE ST; Formal analysis, Methodology, Supervision, Funding acquisition: Lee ST; Writing-original draft: Jang Y, Lee ST; Writing-review and editing: all authors.

\section{REFERENCES}

1. Graus F, Titulaer MJ, Balu R, et al. A clinical approach to diagnosis of autoimmune encephalitis. Lancet Neurol 2016;15:391-404.

2. Graus F, Delattre JY, Antoine JC, et al. Recommended diagnostic crite- ria for paraneoplastic neurological syndromes. J Neurol Neurosurg Psychiatry 2004;75:1135-1140.

3. Dalmau J, Graus F. Antibody-mediated encephalitis. N Engl J Med 2018;378:840-851.

4. Ney DE, Messersmith W, Behbakht K. Anti-ma2 paraneoplastic encephalitis in association with recurrent cervical cancer. J Clin Neurol 2014;10:262-266.

5. Longo R, Wagner M, Savenkoff B, et al. A paraneoplastic limbic encephalitis from an anorectal small cell neuroendocrine carcinoma: a case report. BMC Neurol 2019;19:304.

6. Jung NY, Woo KN, Cho JW, Kim HW. Anti-Yo-associated autoimmune encephalitis after colon cancer treatment. J Neurocrit Care 2020;13: 104-107.

7. Gultekin SH, Rosenfeld MR, Voltz R, Eichen J, Posner JB, Dalmau J. Paraneoplastic limbic encephalitis: neurological symptoms, immunological findings and tumour association in 50 patients. Brain 2000; 123(Pt 7):1481-1494.

8. Patard JJ, Leray E, Rioux-Leclercq N, et al. Prognostic value of histologic subtypes in renal cell carcinoma: a multicenter experience. J Clin Oncol 2005;23:2763-2771.

9. Bell BB, Tognoni PG, Bihrle R. Limbic encephalitis as a paraneoplastic manifestation of renal cell carcinoma. J Urol 1998;160(3 Pt 1):828.

10. Harrison JW, Cherukuri R, Buchan D. Renal cell carcinoma presenting with paraneoplastic hallucinations and cognitive decline from limbic encephalitis. J Gen Intern Med 2015;30:1037-1040.

11. Zhu L, Deng X, Lai Z, Xie N, Tan W. Paraneoplastic limbic encephalitis cured with nephron-sparing surgery in a patient with clear cell renal cell carcinoma: a case report. J Int Med Res 2019;47:5318-5322.

12. Jang Y, Kim DW, Yang KI, et al. Clinical approach to autoimmune epilepsy. J Clin Neurol 2020;16:519-529.

13. Lim JA, Lee ST, Moon J, et al. Development of the clinical assessment scale in autoimmune encephalitis. Ann Neurol 2019;85:352-358.

14. Davis CF, Ricketts CJ, Wang M, et al. The somatic genomic landscape of chromophobe renal cell carcinoma. Cancer Cell 2014;26:319-330.

15. Cancer Genome Atlas Research Network; Linehan WM, Spellman PT, et al. Comprehensive molecular characterization of papillary renal-cell carcinoma. N Engl J Med 2016;374:135-145. 